\title{
ON THE CLOSURE OF CERTAIN BANACH SPACES OF FUNCTIONS OF SEVERAL VARIABLES
}

\author{
BY DAVID A. SPRECHER ${ }^{1}$
}

Communicated by L. Nirenberg, January 13, 1967

1. Statement of the main results. Our primary goal in this note is to establish the following proposition.

ThEOREM 1. Consider the Banach space $\mathfrak{C}_{n}$ of continuous real-valued functions $f: E_{n} \rightarrow R, E_{n}$ standing for the unit cube in n-dimensional Euclidean space. If $\phi$ and $\psi$ are any fixed functions of $\mathfrak{C}_{n}$ with connected level-sets intersecting pairwise in connected sets, then the subspace of superpositions $a \circ \phi+b \circ \psi$ is closed in $\mathfrak{C}_{n}$ under the uniform norm.

Mark by $\beta_{n}$ the indicated space of superpositions. To prove the stated theorem, it suffices to verify

THEOREM 2. Every function of $\mathfrak{C}_{n}$ has a best uniform approximation in $\beta_{n}$.

Distinguish one of the fixed functions, say, $\psi$; denote its level sets by $l_{\psi}(t)$,

$$
l_{\psi}(t)=\left\{\boldsymbol{p} \in E_{n}: \psi(p)=t\right\}
$$

designate by $L_{\psi}$ the aggregate of level sets $l_{\psi}=l_{\psi}(t)$. Finally, set for each $f \in \mathfrak{e}_{n}$

$$
\begin{aligned}
\omega\left(f \mid l_{\psi}\right) & =\max _{p \in l} f(p)-\min _{p \in l_{\psi}} f(p), \\
\omega(f \mid \psi) & =\max _{l_{\psi} \in L_{\psi}} \omega\left(f \mid l_{\psi}\right), \\
\mu(f) & =\inf _{\mathbb{B}_{n}}\|f-a \circ \phi-b \circ \psi\|,
\end{aligned}
$$

(the properties of the functional $\omega$ and related topics are investigated in [1] and [2]. Theorem 2 is proved by means of the five lemmas now formulated.

Lemma 1. For each $f \in \mathbb{C}_{n}$,

$$
\mu(f)=\frac{1}{2} \inf _{a \in \mathfrak{e}} \omega(f-a \circ \phi \mid \psi),
$$

1 This research was supported in part by the National Science Foundation under Grant No. GP-4165. 
where $\mathcal{C}$ stands for the appropriate space of continuous functions of a single variable.

Lemma 2. The function $\alpha \circ \phi+\beta \circ \psi$ of $\Theta_{n}$ is a best approximation to $f \in \mathcal{C}_{n}$ if $\alpha$ minimizes the functional $\omega$,

$$
\omega(f-\alpha \circ \phi \mid \psi)=\inf _{a \in \mathfrak{e}} \omega(f-a \circ \phi \mid \psi),
$$

and $\beta \circ \psi$ is defined for each $l_{\psi}$ as

$$
\beta o \psi=\frac{1}{2}\left[\max _{\imath \psi}(f-\alpha o \phi)+\min _{\iota \psi}(f-\alpha o \phi) .\right.
$$

Since $\omega$ measures the oscillation of $f$ on the partition $L_{\psi}$ (of $E_{n}$ ), we agree to call $\alpha$ a best $\omega$-approximation of $f$ (see also [3]).

LEMma 3. Given a function $f \in \mathrm{e}_{n}$, consider the family $\mathfrak{f}$ whose members are defined for each admitted level set $l_{\psi}(t)$ to be the restriction of $f$ to it:

$$
\mathfrak{f}=\left\{f_{t}: f_{t}=f \mid l_{\psi}(t)\right\}
$$

let $\mathfrak{f}_{\epsilon} \subset \mathfrak{f}$ be an $\epsilon$-net of the family $\mathfrak{f}$, then

$$
\inf _{a \in \mathfrak{e}} \omega\left(\mathfrak{f}_{\bullet}-a \circ \phi \mid \psi\right) \leqq \mu(f) \leqq \inf _{a \in \mathfrak{e}} \omega\left(\mathfrak{f}_{e}-a \circ \phi \mid \psi\right)+\epsilon,
$$

where

$$
\omega\left(\mathfrak{f}_{\epsilon}-a \circ \phi \mid \psi\right)=\max _{f_{t} \in \mathfrak{f}_{\epsilon}} \omega\left(f_{t}-a \circ \phi \mid l_{\psi}(t)\right) .
$$

LEMMA 4. Each finite family $\mathfrak{f}_{\mathrm{\epsilon}}$ has a best $\omega$-approximation.

Lemma 5. Corresponding to the members $\epsilon_{k}>0$ of the convergent series $\sum \epsilon_{k}$, let $\left\{\mathfrak{f}_{k}\right\}$ be an ascending chain of $\epsilon_{k}$-nets of $\mathfrak{f}$ :

$$
\mathfrak{f}_{1} \subset \mathfrak{f}_{2} \subset \cdots \subset \mathfrak{f}_{k} \subset \cdots \subset \mathfrak{f} .
$$

There is a uniformly convergent sequence of best $\omega$-approximations $\alpha_{k}$ to $\mathfrak{f}_{k}$ whose limit is a best $\omega$-approximation to the function $f$ attached to $\mathfrak{f}_{\text {. }}$

Theorem 1 was arrived at through an examination of the constructions in [2]. Specifically, the first three lemmas above correspond to the first three theorems in [2]; Lemma 5 is the counterpart of [2, Theorem 4]. The proofs of the preceding lemmas require certain modifications of the arguments in [2], based on these facts:

Given $f$, let $\mathfrak{f}_{\epsilon}=\left\{f_{1}, \cdots, f_{m}\right\}, f_{j}=f \mid l_{\psi}\left(t_{j}\right)$, be a fixed $\epsilon$-net of $\mathfrak{f}$; let $\mathfrak{f}_{e k}, 1 \leqq k \leqq m$, consist of the first $k$ elements of $\mathfrak{f}_{\mathrm{e}}$.

(i) If $\alpha_{k}$ is a best $\omega$-approximation of $\mathrm{f}_{e k}$, then so is $\alpha_{k}+c$ for each constant $c$. 
(ii) If $\alpha_{k+1}$ is a best $\omega$-approximation of $\mathrm{f}_{\epsilon k+1}$, then there is a constant $c$ such that for each point $\boldsymbol{p} \in l_{\psi}\left(t_{k+1}\right), \operatorname{sgn}\left[f_{k+1}-\alpha_{k+1} \circ \phi-c\right]$ $=\operatorname{sgn}\left[\alpha_{k+1} \circ \phi+c-\alpha_{k} \circ \phi\right]$, unless $f_{k+1}=\alpha_{k+1} \circ \phi+c$ or $\alpha_{k} \circ \phi=\alpha_{k+1} \circ \phi$ $+c$.

2. On the choice of $\phi$ and $\psi$. The following propositions are conveniently formulated with this notation:

Consider two functions, $\psi_{1}$ and $\psi_{2}$, of $\mathfrak{e}_{n}$ : if $L_{\psi_{1}}$ is a refinement of $L_{\psi_{2}}$, when these are regarded as partitions of $E_{n}$, then we write $\psi_{2} \succ \psi_{1}$ (see [1]). Designating now best approximations of $f$ in $\Theta_{n}$ relative to the fixed functions $\phi$ and $\psi$ by $\mu(f ; \phi, \psi)$, we state

TheOREM 3. If $\phi_{2} \succ \phi_{1}$ and $\psi_{2} \succ \psi_{1}$, then

$$
\mu\left(f ; \phi_{2}, \psi_{2}\right) \geqq \mu\left(f ; \phi_{1}, \psi_{1}\right)
$$

for each $f \in \mathfrak{C}_{n}$.

THEOREM 4. If $\phi \succ \psi$, then

$$
\mu(f ; \phi, \psi)=\frac{1}{2} \omega(f \mid \psi) .
$$

ThEOREM 5. For each $p \in E_{n}$ and $i=1,2$, let $U_{\text {ip }}$ designate the intersection of level sets $l_{\phi_{i}}$ and $l_{\psi_{i}}$ containing $p$ (that is, we remove now the restriction imposed in the fixed functions in Theorem 1). If $U_{2 p} \supset U_{1 p}$ for each $p \in E_{n}$, then the conclusion of Theorem 3 remains valid for each $f \in \mathcal{C}_{n}$.

Theorem 3 is a generalization of $[1$, Corollary 3.2$]$. To prove it, consider first the case when $\phi_{1}=\phi_{2}$. Given $f$, let $\left\{a_{k}\right\}$ be a sequence of members of $\mathfrak{e}$ such that

$$
\lim _{\boldsymbol{k} \rightarrow \infty} \omega\left(f-a_{k} \circ \phi_{2} \mid \psi_{2}\right)=\mu\left(f ; \phi_{2}, \psi_{2}\right):
$$

clearly, we are guaranteed the existence of such sequences. Owing to $[1$, Corollary 2.1$]$, we have the inequality

$$
\omega\left(f-a_{k} \circ \phi_{2} \mid \psi_{2}\right) \geqq \omega\left(f-a_{k} \circ \phi_{2} \mid \psi_{1}\right)
$$

for each fixed $k$, and consequently this remains true as we let $k \rightarrow \infty$; while we are not assured the convergence of the sequence whose members are $\omega\left(f-a_{k} \circ \phi_{2} \mid \psi_{1}\right)$, we can state that

$$
\mu\left(f ; \phi_{2}, \psi_{2}\right) \geqq \limsup _{x \rightarrow \infty} \omega\left(f-a_{k} \circ \phi_{2} \mid \psi_{1}\right) \text {. }
$$

If it happens that the right side of this inequality equals $\mu\left(f ; \phi_{2}, \psi_{1}\right)$ then we have established this case; otherwise, we consider a sequence, $\left\{c_{k}\right\}$, of functions $c_{k} \in \mathfrak{e}$, subject to the specification 


$$
\lim _{x \rightarrow \infty} \omega\left(f-c_{k} \circ \phi_{2} \mid \psi_{1}\right)=\mu\left(f ; \phi_{2}, \psi_{1}\right) .
$$

A comparison of the last two inequalities reveals at once that

$$
\mu\left(f ; \phi_{2}, \psi_{2}\right) \geqq \mu\left(f ; \phi_{2}, \psi_{1}\right),
$$

thereby disposing of the case in question.

The proof of the theorem is completed by applying Lemma 1 above to the last inequality, and using the fact that

$\mu(f, \phi, \psi)=\inf _{a \in \mathfrak{e}} \inf _{b \in \mathfrak{e}}\|f-a \circ \phi-b \circ \psi\|=\inf _{b \in \mathfrak{e}} \inf _{a \in \mathfrak{e}}\|f-a \circ \phi-b \circ \psi\|$.

Theorem 4 follows at once from the fact that under these circumstances $\phi=g \circ \psi$ for some function $g \in \mathcal{C}$ (see [1]).

Theorem 5 is verified with the basic inequality $\omega(f \mid S) \geqq \omega(f \mid T)$ whenever $S \supset T$ and the fact that if $l_{\phi} \cap l_{\psi}=S$, then

$$
\mu(f \mid S ; \phi, \psi)=\frac{1}{2} \omega(f \mid S) .
$$

In particular, this theorem implies that in order to optimize our best approximations, all members of $L_{\phi}$ should intersect those of $L_{\psi}$ in at most one point.

In connection with these results we pose this problem:

It is well known that certain partitions of $E_{n}$ cannot qualify for membership in the family of level sets of a continuous function. Thus, for example, when $L_{f}=E_{n}$, then $f$ is at most continuous almost everywhere. We ask, therefore, for necessary and sufficient conditions for a partition $P$ of $E_{n}$ to admit a function $f \in \mathcal{C}_{n}$, such that $P=L_{f}$; similarly, under what circumstances can the function so associated with $P$ be continuous merely almost everywhere?

\section{BIBLIOGRAPHY}

1. D. A. Sprecher, On best approximations in several variables, J. Reine Angew. Math (to appear).

2. - On best approximations of functions of two variables, (to appear).

University of California, Santa Barbara 